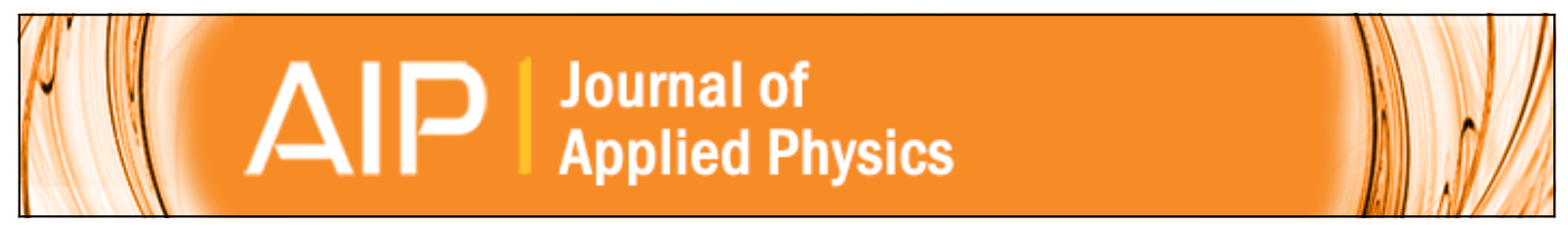

\title{
Hybridization-induced magnetism in correlated cerium systems
}

Nicholas Kioussis, J. Thevenot, Bernard R. Cooper, and Q. G. Sheng

Citation: Journal of Applied Physics 79, 6420 (1996); doi: 10.1063/1.361957

View online: http://dx.doi.org/10.1063/1.361957

View Table of Contents: http://scitation.aip.org/content/aip/journal/jap/79/8?ver=pdfcov

Published by the AIP Publishing

\section{Articles you may be interested in}

Strongly Correlated Electron Behaviors and Heavy Fermions in Anomalous Rare-earth and actinide Systems AIP Conf. Proc. 846, 3 (2006); 10.1063/1.2222266

A hybrid laser system consisting of a frequency-doubled, narrow-line-width, distributed-feedback dye laser oscillator and a high saturation-fluence Ce:LiCaAIF 6 crystal amplifier

Appl. Phys. Lett. 82, 3391 (2003); 10.1063/1.1576294

Absolute evaluation of combined hybridization-induced and RKKY-induced two-ion interaction in correlated electron systems

J. Appl. Phys. 69, 5472 (1991); 10.1063/1.347995

Trends of hybridization-induced magnetism in cerium monochalcogenides

J. Appl. Phys. 69, 5475 (1991); 10.1063/1.347970

Theory of strong hybridization-induced relaxation in uranium systems

J. Appl. Phys. 64, 5592 (1988); 10.1063/1.342291

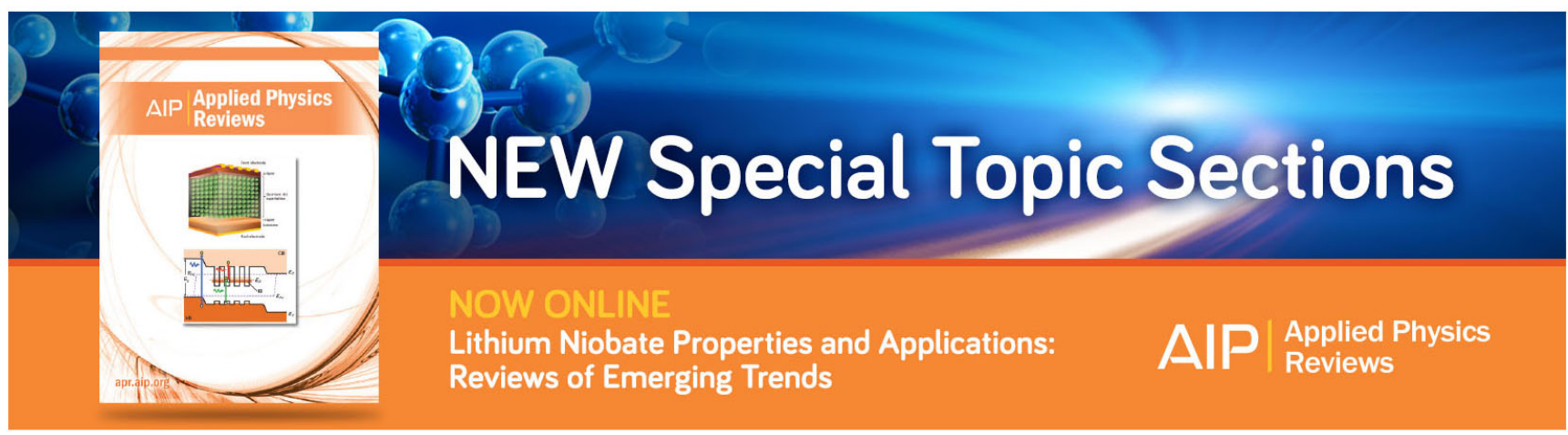




\title{
Hybridization-induced magnetism in correlated cerium systems
}

\author{
Nicholas Kioussis and J. Thevenot \\ Department of Physics and Astronomy, California State University, Northridge, California 91330-8268
}

Bernard R. Cooper and Q. G. Sheng

Department of Physics, West Virginia University, Morgantown, West Virginia 26506

\begin{abstract}
There is a great change in the nature of the magnetic ordering on going from $\mathrm{CeIn}_{3}$, a local moment antiferromagnetic system, to $\mathrm{CePb}_{3}$, a heavy fermion itinerant antiferromagnetic system, both of which have $\mathrm{Cu}_{3} \mathrm{Au}$ crystal structure. We have applied ab initio electronic structure calculations, based on the linear-muffin-tin-orbital method, and a phenomenological theory of orbitally driven magnetic ordering, to study the effects of the band- $f$ hybridization-induced interactions and the band- $f$ exchange-induced interactions, pertinent to the magnetic behavior of these systems. The position of the Ce $4 f$ energy level relative to the Fermi energy and the intra-atomic Coulomb interaction are obtained from a sequence of three total-energy supercell calculations with two, one and zero $f$ electrons in the $\mathrm{Ce} 4 f$ core. The calculations elucidate the origins in the electronic structure of the variation of the $f$-state resonance width characterizing the strength of the hybridization and the density of states at the Fermi energy characterizing the number and character of band states available for hybridization. We present results for the hybridization potential and the hybridization-induced exchange interactions on going from $\mathrm{CeIn}_{3}$ to $\mathrm{CePb}_{3}$, where the only obvious change is the addition of an anion $p$ electron. (C) 1996 American Institute of Physics.
\end{abstract} [S0021-8979(96)26608-0]

\section{INTRODUCTION}

The $\mathrm{CeX}_{3}$ compounds with $\mathrm{X}=\mathrm{In}, \mathrm{Sn}$, and $\mathrm{Pb}$ provide the opportunity to study the competition between different ground states in cerium systems. ${ }^{1}$ These compounds are all cubic with the $\mathrm{Cu}_{3} \mathrm{Au}$ structure. $\mathrm{CeIn}_{3}$ is a local moment antiferromagnetic system ${ }^{2}$ (LMAF) with a Néel temperature of about $10 \mathrm{~K}$ and a weak low-temperature moment of $0.4 \mu_{B}$ that shows strong Kondo resistivity. ${ }^{3}$ The cerium moments are aligned antiferromagnetically in adjacent (111) ferromagnetic planes. When a small amount of tin is substituted for indium, the magnetic ground state is no longer stable, and the system no longer orders. ${ }^{1}$ This indicates that the underlying energy parameters in $\mathrm{CeIn}_{3}$ lie near the values at which a magnetic-nonmagnetic instability of the $4 f$ electron occurs. $\mathrm{CeSn}_{3}$ is a mixed valent (MV) system ${ }^{1}$ which does not order magnetically. Finally, $\mathrm{CePb}_{3}$ has been shown ${ }^{4}$ to be an itinerant antiferromagnet with an extremely small moment and a Néel temperature of $1.2 \mathrm{~K}$. Moreover, several $\mathrm{CePb}_{3}$ properties show heavy fermion-like behavior. ${ }^{4}$

The purpose of the work reported here was to investigate the origins in the electronic structure, of the variation of the $f$-state resonance width characterizing the strength of the hybridization, the density of states at the Fermi energy, characterizing the number and character of band states available for hybridization, the hybridization potential, and the hybridization-induced and exchange-induced interactions on going from $\mathrm{CeIn}_{3}$ to $\mathrm{CePb}_{3}$, where the only obvious change is the addition of an anion $p$ electron. We have employed a unified theory that we have recently developed, ${ }^{5-11}$ which involves a synthesis of (i) a phenomenological theory of moderately delocalized $f$-electron systems which includes explicit correlation effects and treats both the band- $f$ hybridization and the band- $f$ Coulomb exchange on an equal footing, and (ii) $a b$ initio electronic structure calculations, based on the linear-muffin-tin-orbital (LMTO) method, ${ }^{12}$ allowing a first-principles evaluation of the parameters entering the phenomenological theory. The interconfigurational correlation effects, which are important for this class of systems and which are treated explicitly in our theory, are not preserved in band theory and cannot be captured by the exchange correlation potential used in band theory.

The self-consistent one-electron potential is obtained from a warped-muffin-tin LMTO calculation without recourse to the atomic sphere approximation, ${ }^{12}$ i.e., using a nonzero tail parameter in the interstitial, which is treated as a variational parameter. Two energy windows are employed to allow coverage of various subbands: the semicore cerium $5 p$ states are calculated with a tail parameter about $-0.9 \mathrm{Ry}$, and the cerium valence $6 s, 6 p$, and $5 d$ states and anion $\operatorname{In}(\mathrm{Pb})$ $5 s(6 s)$ and $5 p(6 p)$ states with a tail parameter, which is the average energy over occupied states in the interstitial. The full potential in the interstitial is used and the only shape approximation to the potential is a spherical averaging in nonoverlapping muffin-tin spheres. The cerium $4 f$ electrons are treated as localized rather than itinerant states and thus they are included self-consistently as core states at each iteration, not being allowed to hybridize with band states. The relative magnitude of the muffin-tin radii are chosen so that nearest-neighbor muffin-tin spheres touch at the minimum in the charge density between nearest neighbors. While the basis functions for the band states are scalar relativistic, ${ }^{13}$ spinorbit coupling is included self-consistently. The electronic structure provides the band states and energies, and a selfconsistent hybridization potential, thereby allowing determination of the band- $f$ hybridization potential matrix elements, $V_{\mathbf{k} m}$ and the band- $f$ coulomb exchange matrix elements $J_{n n^{\prime}}\left(\mathbf{k}, \mathbf{k}^{\prime}\right)$. The $4 f$-state energy relative to the Fermi energy $E_{f}-E_{F}$, and the intra-atomic correlation energy $U$, are determined from differences of total energy calculations for different $f$ configurations as impurities in a supercell 


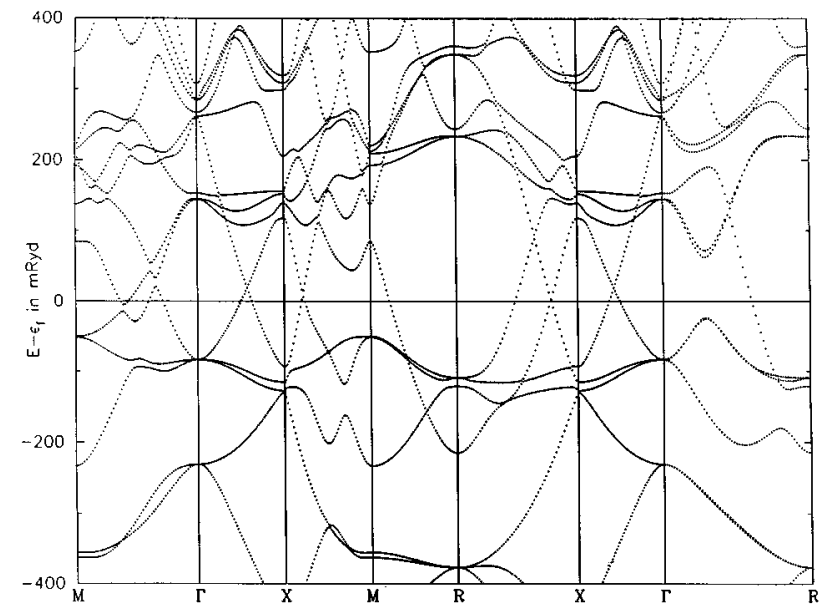

FIG. 1. The band structure of $\mathrm{CeIn}_{3}$, calculated with the cerium $4 f$ states treated as core states, along symmetry lines in the Brillouin zone. The band energies are with respect to the Fermi energy.

$\left(f^{0}, f^{1}, f^{2}\right)$ where all cerium sites but one have the nominal configuration $f^{1}$. Having determined the phenomenological model Hamiltonian parameters on an absolute basis, one can evaluate $^{6-11}$ the hybridization-induced and RKKY (but with the orbital part included) two-ion exchange interactions pertinent to the unusual magnetic ordering in this class of systems.

\section{RESULTS AND DISCUSSION}

The band structure of $\mathrm{CeIn}_{3}$ (typical of this class of systems), calculated with the Ce $4 f$ states treated as core states, is plotted along symmetry lines in Fig. 1, with the band energies measured with respect to the Fermi energy. We treat the effects of band- $f$ hybridization within the context of the Anderson model Hamiltonian, where the hybridization matrix element $V_{k m}$ in the model Hamiltonian is taken to be the matrix element of the muffin-tin Hamiltonian between band states of non- $f$ character and the wave function representing the $\mathrm{Ce} 4 f_{5 / 2}$ state, $\psi_{f}$, which is obtained (self-consistently) from the resonance in the potential within the Ce muffin tin. ${ }^{6,7}$ The bands dominating the hybridization-induced properties are those lying near the Fermi energy, which are largely derived from anion $p$-derived and cerium $d$-derived bands. Within the context of the model Hamiltonian, anion derived $p$-states hybridize more strongly with the Ce $4 f$ resonance states, because the $\mathrm{Ce}$-anion separation is smaller than the $\mathrm{Ce}-\mathrm{Ce}$ separation. The bands at $\Gamma$ which are 85 mRy below the Fermi energy are indium $p$ bands, whereas those which are $144 \mathrm{mRy}$ above the Fermi energy are Ce $d$ bands. The addition of an anion $p$ electron on going from $\mathrm{CeIn}_{3}$ to $\mathrm{CePb}_{3}$ causes the anion $p$-derived bands at $\Gamma$ to shift at $189 \mathrm{mRy}$ below the Fermi energy, thus suppressing the $p$ - $f$ hybridization. This is consistent with the decrease of the $f$-state resonance width listed in Table I, characterizing the strength of hybridization. The partial density of states (DOS) corresponding to the band structure in Fig. 1 is shown in Fig. 2. Plotted are the partial DOS for the Ce $d$-derived (solid curve), the In $s$-derived (dashed curve), and $p$-derived (dot-
TABLE I. Summary of LMTO results for the Fermi energy $E_{F}$, the Ce $4 f_{5 / 2}$ resonance energy, the total density of states $N\left(E_{F}\right)$ at $E_{F}$, the Ce $d$-derived and anion (In or $\mathrm{Pb}$ ) $p$-derived partial density of states at $E_{F}$, the $\mathrm{Ce} 4 f_{5 / 2}$ resonance width $\Gamma$, and the hybridization potential $v\left(\kappa_{F}\right)$, for $\mathrm{CeIn}_{3}$ and $\mathrm{CePb}_{3}$, respectively. Also listed are the results of the supercell LMTO calculations for the position of the $f$ state relative to the $E_{F}$, and the intraatomic Coulomb interaction $U$.

\begin{tabular}{lcc}
\hline \hline & $\mathrm{CeIn}_{3}$ & $\mathrm{CePb}_{3}$ \\
\hline Lattice constant (a.u.) & 8.859 & 9.210 \\
Ce muffin-tin radius (a.u.) & 2.975 & 3.100 \\
Anion muffin-tin radius (a.u.) & 3.132 & 3.256 \\
$E_{F}(\mathrm{Ry})$ & 0.487 & 0.453 \\
$\mathrm{Ce} 4 f$ resonance energy (Ry) & 0.503 & 0.442 \\
$N\left(E_{F}\right)\left(\mathrm{Ry}^{-1}\right)$ & 27.3 & 41.5 \\
$N_{d}^{\mathrm{Ce}}\left(E_{F}\right)\left(\mathrm{Ry}^{-1}\right)$ & 5.5 & 7.5 \\
$N_{p}^{\text {anion }}\left(E_{F}\right)\left(\mathrm{Ry}^{-1}\right)$ & 12.3 & 23.3 \\
$\Gamma(\mathrm{mRy})$ & 6.30 & 5.13 \\
$v\left(\kappa_{F}\right)(\mathrm{mRy})$ & -2.55 & -2.21 \\
$E_{f}-E_{F}(\mathrm{eV})$ & -3.2 & -3.0 \\
$U(\mathrm{eV})$ & 6.2 & 6.3 \\
\hline \hline
\end{tabular}

ted curve) bands, respectively. The Fermi energy $E_{F}$, and the DOS at the Fermi energy $N\left(E_{F}\right)$, in $\mathrm{CeIn}_{3}$ are $0.487 \mathrm{Ry}$ and 27.3 states/Ry, respectively, compared to the corresponding values of $0.453 \mathrm{Ry}$ and 41.5 states/Ry in $\mathrm{CePb}_{3}$. On going to $\mathrm{CePb}_{3}$ there is an opening of a gap in the DOS between -0.025 and $0.18 \mathrm{Ry}$, and there is an increase (by a factor of 2 ) in the anion $p$-projected density of states at the Fermi energy.

For the purpose of analyzing hybridization effects within the context of our procedure for calculating Anderson model Hamiltonian parameters, band- $f$ hybridization in these compounds may be approximately characterized by two quantities arising from our band structure calculations: $f$-state resonance widths, characterizing the strength of hybridization, and the density of states at the Fermi energy, characterizing the number and character of bands available for

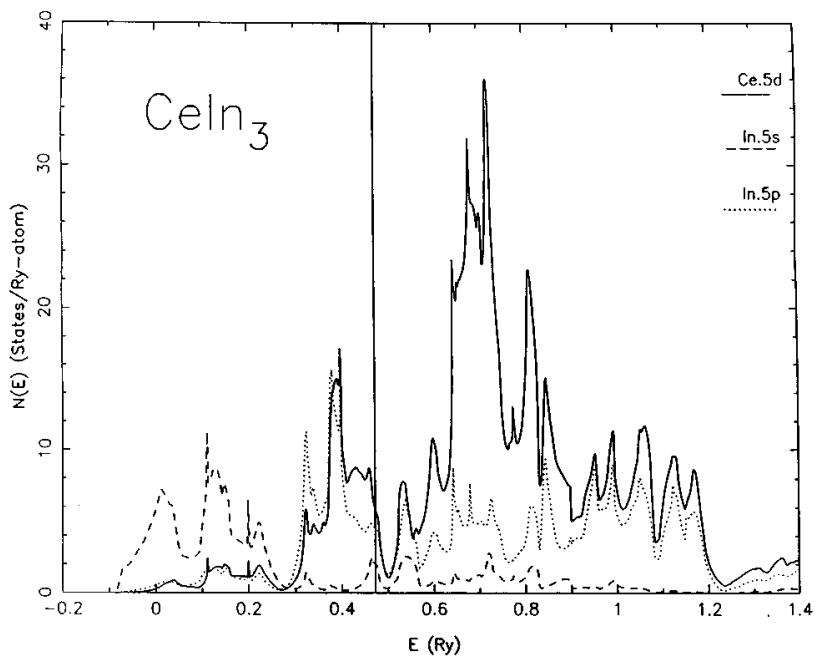

FIG. 2. Calculated partial density of states of $\mathrm{CeIn}_{3}$ corresponding to the band structure of Fig. 1. Plotted are the density of states for the Ce $d$-derived (solid curve), the In $s$-derived (dashed curve), and $p$-derived (dotted curve) bands, respectively. 
hybridization. ${ }^{6,7}$ Calculated results of the cerium and anion muffin-tin radius, the Fermi energy, the cerium $4 f$ resonant energy, the total and partial cerium $d$ - and anion $p$-derived density of states at $E_{F}$, the $f$-state resonance width $\Gamma$, and the hybridization potential $v\left(\kappa_{F}\right)$, are listed in Table I, for $\mathrm{CeIn}_{3}$ and $\mathrm{CePb}_{3}$, respectively. The density of states at $E_{F}$, $N\left(E_{F}\right)$, are largely derived from anion $p$ states and cerium $5 d$ states. It is important to note that the values of $\Gamma$ for both $\mathrm{CeIn}_{3}$ and $\mathrm{CePb}_{3}$ are comparable to that calculated ${ }^{7}$ for $\mathrm{CeTe}$ $(\Gamma=5.7 \mathrm{mRy})$, which was found to be at the borderline between magnetic and nonmagnetic behavior in contrast to the almost fully magnetically ordered CeSb. While the total density of states available for hybridization increases on going from $\mathrm{CeIn}_{3}$ to $\mathrm{CePb}_{3}$, the resonance width decreases because of the larger Ce-anion separation in $\mathrm{CePb}_{3}$.

The energy necessary to place the Ce $f$-electron in a band state at the Fermi energy, $E_{F}-E_{f}$, and the energy required to change from an $f^{1}$ configuration to an $f^{2}$ configuration, $E_{f}+U-E_{F}$, have been evaluated for both systems. The values of $E_{F}-E_{f}$ and $U$ are then obtained from the $f^{0}$, $f^{1}$, and $f^{2} f$-state eigenvalues by the use ${ }^{7}$ of linear transition theory. We find that $E_{F}-E_{f}$ and $U$ are 3.2 and $6.2 \mathrm{eV}$ in $\mathrm{CeIn}_{3}$, and 3.0 and $6.3 \mathrm{eV}$ in $\mathrm{CePb}_{3}$, respectively. We have also calculated the $6 \times 6$ hybridization-induced exchange interaction matrix, $E_{n}=E\left(m, m^{\prime}, \mathbf{R}_{n}\right),{ }^{7}$ pertinent to the magnetic behavior of this class of systems. We find that the dominant matrix elements involve the $m=m^{\prime}= \pm 1 / 2$ states, which correspond to the piling up of charge along the interionic axis. $E_{1}$, which in the phenomenological treatment, ${ }^{5}$ is chosen to match the Néel temperature, is about the same in $\mathrm{CeIn}_{3}(2.9 \mathrm{~K})$ and in $\mathrm{CePb}_{3}(3.0 \mathrm{~K})$. More importantly, we find that $E_{2}\left(E_{3}\right)$ are ferromagnetic and large in both compounds, $3.1 \mathrm{~K}(2.9 \mathrm{~K})$ in $\mathrm{CeIn}_{3}$ and $2.7 \mathrm{~K}(2.8 \mathrm{~K})$ in $\mathrm{CePb}_{3}$. As for the case of other cerium systems (CeSb,CeTe), Coulomb exchange rather than hybridization was found ${ }^{10}$ to be the larger contributor to the highly anisotropic effective twoion interaction which determines the magnetic ordering be- havior. Work currently in progress is aimed at the evaluation of the Coulomb exchange-induced interactions (proportional to $\left.J^{2}\right){ }^{10,11}$ Having determined the two-ion exchange interactions, the equilibrium magnetic behavior (i.e., the ordered moment and the ordered temperature) of a given magnetic structure will be determined by treating the exchange Hamiltonian [Eq. (3) in Ref. 7] within mean-field theory.

\section{ACKNOWLEDGMENTS}

The research at California State University Northridge (CSUN) was supported through the NSF under Grant No. DMR-89-18887 and the Office of Research and Sponsored Projects at CSUN. The research at West Virginia University was supported through the NSF under Grant No. DMR-9120333.

${ }^{1}$ S. Rahman, J. Timlin, J. E. Crow, T. Mihalisin, and P. Schlottmann, J. Appl. Phys. 67, 5209 (1990).

${ }^{2}$ J. Lawrence and S. M. Shapiro, Phys. Rev. B 22, 4379 (1980).

${ }^{3}$ A. Maury, R. Freitag, J. E. Crow, T. Mihalisin, and A. I. Abou-Aly, Phys. Lett. A 92, 441 (1982).

${ }^{4}$ C. L. Lin, J. Teter, J. E. Crow, T. Mihalisin, J. Brooks, A. I. Abou-Aly, and G. R. Stewart, Phys. Rev. Lett. 54, 2541 (1985); C. Vettier, P. Mortin, and J. Flouquet, ibid. 56, 1980 (1986).

${ }^{5}$ B. R. Cooper, R. Siemann, D. Yang, P. Thayamballi, and A. Banerjea, in Handbook on the Physics and Chemistry of the Actinides, edited by A. J. Freeman and G. H. Lander (North Holland, Amsterdam, 1985), Chap. 6, pp. 435-500.

${ }^{6}$ J. M. Wills and B. R. Cooper, Phys. Rev. B 36, 3809 (1987).

${ }^{7}$ N. Kioussis, B. R. Cooper, and J. M. Wills, Phys. Rev. B 44, 10003 (1991)

${ }^{8}$ N. Kioussis, H. J. Yu, B. R. Cooper, Q. G. Sheng, and J. M. Wills, J. Appl. Phys. 73, 5424 (1993).

${ }^{9}$ B. R. Cooper, Q. G. Sheng, S. P. Lim, C. Sanchez-Castro, N. Kioussis, and J. M. Wills, J. Magn. Magn. Mater. 108, 10 (1992).

${ }^{10}$ Q. G. Sheng and B. R. Cooper, Phys. Rev. B 50, 965 (1994); J. Appl. Phys. 69, 5472 (1991).

${ }^{11}$ Q. G. Sheng, B. R. Cooper, and S. P. Lim, Phys. Rev. 50, 9215 (1994); J. Appl. Phys. 70, 6083 (1991).

${ }^{12}$ O. K. Andersen, Phys. Rev. B 12, 3060 (1975).

${ }^{13}$ D. D. Koelling and B. N. Harmon, J. Phys. C 10, 3107 (1977). 